\title{
Efficiency of DCT-Based Denoising Techniques Applied to Texture Images
}

\author{
Aleksey Rubel ${ }^{1}$, Vladimir Lukin ${ }^{1}$, and Oleksiy Pogrebnyak ${ }^{2}$ \\ ${ }^{1}$ National Aerospace University named by N.Y. Zhukovsky, Department of Receivers, \\ Transmitters and Signal Processing, Chkalova St. 17, 61070, Kharkov, Ukraine \\ edu.rubel@gmail.com, vladimlukin@yahoo.com \\ ${ }^{2}$ Instituto Politecnico Nacional, Centro de Investigacion en Computacion, Ave. Juan de Dios \\ Batiz S/N, C.P. 07738, Mexico, D.F., Mexico \\ olek@pollux.cic.ipn.mx
}

\begin{abstract}
Textures or high-detailed structures contain information that can be exploited in pattern recognition and classification. If an acquired image is noisy, noise removal becomes an operation to improve image quality before further stages of processing. Among possible variants of denoising, we consider filters based on orthogonal transforms, in particular, on discrete cosine transform (DCT) known to be able to effectively remove additive white Gaussian noise (AWGN). Besides, we study a representative of nonlocal denoising techniques, namely, BM3D known as state-of-the-art technique based on DCT and similar patch search. We show that noise removal in texture images using the considered DCT-based techniques can distort fine texture details. To detect such situations and avoid texture degradation due to filtering, we propose to apply filtering efficiency prediction tests applicable to wide class of images. These tests are based on DCT coefficient statistic parameters and can be used for decision-making in relation to the use of the considered filters.
\end{abstract}

Keywords: denoising, filter, texture images, DCT, BM3D, AWGN, statistics, visual quality.

\section{Introduction}

Texture features of images or their fragments are widely exploited in numerous applications of pattern recognition [1], remote sensing [2], similarity search in large databases [3]. Often, in such applications noise is present in acquired (original) images. In fact, noise might be the most destructive factor for solving of all related practical problems. For this reason, the noisy data must be analyzed concerning decisionmaking in the followed processing [1-5]. Actually, for texture images the negative influence of noise might occur more apparently than for images with simpler structure. Fine texture details can be essentially disguised by the noise. Therefore, denoising becomes a desired stage in image processing chain. However, alongside with positive effect of noise removal, denoising can distort images in smaller or larger extent. Hence, it should be performed carefully, especially for texture images. 
Rather efficient denoising techniques have been proposed recently [4-8]. First, filters based on orthogonal transforms have demonstrated good performance in additive white Gaussian noise (AWGN) removal [9]. One of such transforms widely used in image processing is the discrete cosine transform (DCT); certainly, wavelet transforms can be used as well [10]. The main reasons for applying DCT are clear. First, DCT has good compactness of signal energy or "sparseness". Second, fast DCT algorithms have been developed and implemented on general-purpose processors [11] and on FPGA architecture [12]. Third, DCT is performed in blocks and, due to this, is well adapted to local structure of any processed image [13].

The latter property of the standard DCT based denoising is useful. But all local approaches operate only on locally distributed spatial data, so-called "blocks" or "patches". This restricts potential efficiency of denoising images with distributed similar data. These types of data can be edges, textures or other singularities that exceed size of the used blocks. Recall that the commonly used block size in DCT-based denoising is $8 \times 8$ pixels $[4,7,13]$.

Nonlocal approaches can perform processing of such image data more efficiently $[5,6,8]$. For these approaches, similar data (patches, blocks) are collected into arrays and processed together, e.g., using some weight function. Provided noise characteristics are known in advance, denoising procedure is a rather simple operation although essential efforts are spent of similar patch search. BM3D filter [8] which is currently state-of-the-art [13,14] employs advantages of both nonlocal and transform domain approaches where DCT is used for data processing in 3D arrays of similar patches.

By excluding small amplitude spectrum components of transformed image data, noise removal is attained on one hand. On the other hand, this operation can delete or distort some image details. Nonlocal denoising approaches have advantages for images with rather large homogenous regions and sharp edges [13, 14]. Meanwhile, nonlocal filters might also run into difficulties for high-frequency data as texture or other complex structure images.

Thus, it could be nice to predict how denoising can affect an image at hand. The paper [14] presents a lot of useful data for preliminary analysis (including results for many standard test images). First, an approach to determining potential limit of filtering efficiency for images corrupted by AWGN is presented for nonlocal approach under condition of available noise-free image. The results show that noise removal in images with large homogenous regions is easy and there are possibilities to further improve filter performance for such images. Meanwhile, denoising of texture images as high-frequency data is hard. The best of existing filters have practically reached the potential limit for textural images (as, e.g., Baboon), especially for the case of intensive noise $[14,15]$. Then, a natural question arises: "Is denoising really needed for a given image?" And this question most often arises just for texture images which are the main subject of our study.

Note that recently an attempt to predict denoising efficiency has been carried out in [16]. It has been shown that simple statistics of DCT coefficients is $8 \times 8$ blocks can serve for predicting a parameter characterizing filtering efficiency under condition that a dependence between them is established (approximated). The dependence obtained in [16] concerns the ratio of the output MSE and AWGN variance and this 
dependence is quite accurate for a set of the standard test images. However, this set did not contain many texture images. Therefore, it is worth paying special attention to texture images with the final goal of decision-making in denoising applying.

The structure of this paper is the following. In Section 2, two DCT-based denoising techniques are briefly discussed. Preliminaries of study (test images, noise characteristics, parameters of filters) are given in Section 3. Practical results of study are presented in Section 4.

\section{DCT-Based Denoising}

As mentioned above, local filters are usually quite simple procedures. Thereby, a used transform has to be a simple procedure, too. The algorithm that performs DCT as matrix multiplying, due to its linearity, is

$$
\begin{gathered}
\mathbf{B}_{\text {trans }}=\mathbf{T} \cdot \mathbf{B}_{\text {spat }} \cdot \mathbf{T}^{T}, \\
\mathbf{B}_{\text {spat }}^{\text {filt }}=\mathbf{T}^{T} \cdot \mathbf{B}_{\text {trans }}^{\text {filt }} \cdot \mathbf{T},
\end{gathered}
$$

where $\mathbf{B}_{\text {spat }}$ is a spatially represented block, $\mathbf{T}$ is a transform matrix, $\mathbf{B}_{\text {trans }}$ is a transformed block, $\mathbf{B}_{\text {spat }}^{\text {filt }}$ is a spatially represented filtered block, $\mathbf{B}_{\text {trans }}^{\text {filt }}$ is a filtered block in the transform domain. Such DCT implementation decreases computational burden and avoids iterative performing DCT in direct way for each block on image. Inverse transform (2) of filtered blocks $\mathbf{B}_{\text {spat }}^{\text {filt }}$ is carried out at once after denoising procedure. This approach can be used in parallel computations for filtering speed-up.

DCT-based denoising is a simple procedure. DCT filter processes data block-wise by hard thresholding in the following manner:

$$
B_{\text {out }}(k, l)=\left\{\begin{array}{c}
B_{\text {in }}(k, l) \leftarrow\left|B_{\text {in }}(k, l)>\beta \cdot \sigma\right| \\
0 \leftarrow\left|B_{\text {in }}(k, l) \leq \beta \cdot \sigma\right|
\end{array}\right.
$$

where $B_{\text {in }}(k, l)$ and $B_{\text {out }}(k, l)$ are input and output blocks, respectively, of the image data transformed by DCT having indices $k, l=0, \ldots, 7, \beta$ is a thresholding parameter, $\sigma$ denotes the standard deviation of the noise that is assumed zero mean AWGN with a priori known standard deviation.

Blocks are taken on whole image with overall overlapping [7, 13]. As it can be seen from (3), the spectrum components that do not exceed assigned pre-estimated threshold provided by noise standard deviation and $\beta$ are excluded. After local denoising operation, the inverse DCT is performed. To get a final filtered value for a given pixel, the filtered values from all blocks that contain a given pixel are averaged. This essentially improves filtering performance compared to non-overlapping blocks. 
Special attention is paid to setting $\beta$. In [18], it is shown that the optimal value of $\beta$ is in the range $2 \ldots 4$. As it has been shown [7], the optimal $\beta$ is equal to about $2.3 \ldots 2.8$ for a wide class of images. In particular, for texture images the optimal $\beta$ values are about $2.3 \ldots 2.5$, but the gain of denoising efficiency is not significant compared to the standard choice. Thus, the DCT filter has a small computational cost and simple realization. BM3D filter [8] works in the same manner taking into account that denoising is simultaneously applied to the group of found similar blocks.

\section{Simulation Preliminaries}

In our studies, eight grayscale test images are used from the texture image database USC-SIPI Image Database [17]. These test images are shown in Fig. 1. As it is seen from Fig. 1, all images have different type and correlation degree of textures. The size of all test images is $512 \times 512$ pixels.

In simulations, AWGN with a required variance has been generated. Ten realizations of the noise have been considered for each test image and noise level. As denoising criterions, the output mean square error $\left(M S E_{\text {out }}\right)$ and PSNR-HVSM [19] have been used. Recall that PSNR-HVS-M is the filtering efficiency criterion that takes into account features of human vision system to describe visual quality of original and processed images more adequately than standard metrics as MSE or PSNR.

Below we consider performance of two DCT-based filters described in Section 2. In addition to results obtained for these filters, two measures of denoising efficiency have been used. First, the approach for predicting potential efficiency of nonlocal filters proposed by P. Chatterjee and P. Milanfar [14] was exploited. This approach assumes that a noise-free image is available. Then, based on similarity search mechanism and nonlocal denoising principles, this approach allows obtaining potential output MSE for a given variance of AWGN. Thus, it is possible to theoretically predict (determine) what can be minimal output MSE and to compare the values obtained for the existing (analyzed) filters to the corresponding limit.

The second (practical) approach [16] is based on the following general idea. There is a known dependence of a parameter $\alpha$ characterizing filtering efficiency on a parameter $\gamma$ able to characterize a noisy image. Then, estimating $\gamma$ for the analyzed image, it becomes possible to determine $\alpha$. Analyzing $\alpha$, it becomes possible, in particular, to decide automatically is it worth to filter a given image or no.

As parameter $\gamma$, it is possible to exploit the probabilities $P_{2 \sigma}$ or $P_{2.7 \sigma}$, where the former is probability that absolute values of AC DCT coefficients in 8x8 blocks do not exceed $2 \sigma$ and the latter is probability that these absolute values exceed $2.7 \sigma$. Note that these probabilities are dependent $\left(P_{2 \sigma}+P_{2.7 \sigma}<1\right)$ and for pure noise $P_{2 \sigma} \approx 0.95$. The ratio $M S E_{\text {out }} / \sigma^{2}$ was used as the parameter $\alpha$ in [14]. In this paper, we also analyze improvement of PSNR-HVS-M (IPSNR-HVS-M, expressed in dB) calculated as difference of PSNR-HVS-M for denoised and original images. 


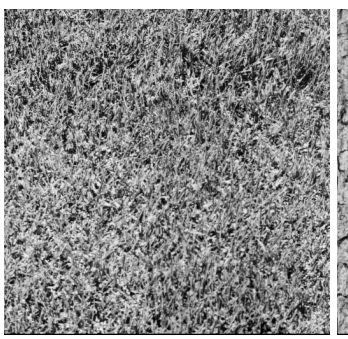

1

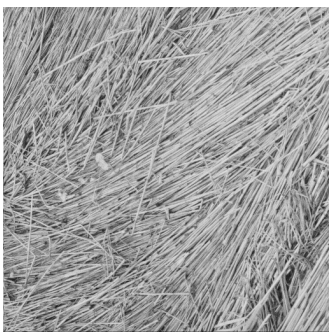

3

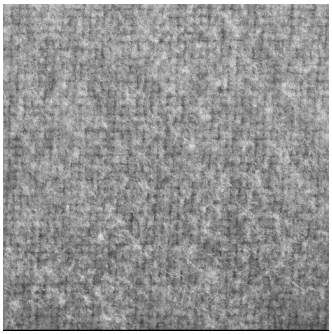

5

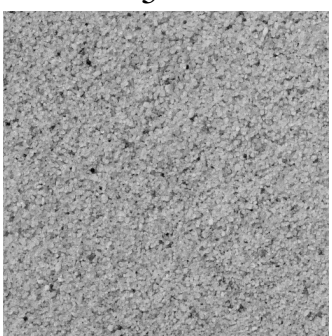

7

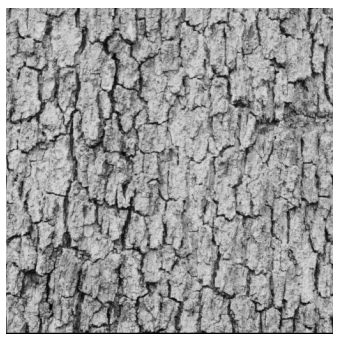

2

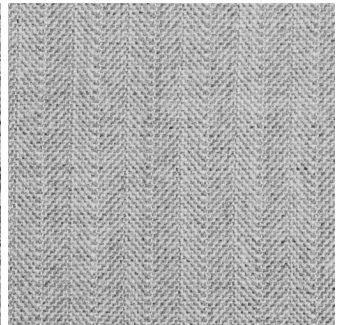

4

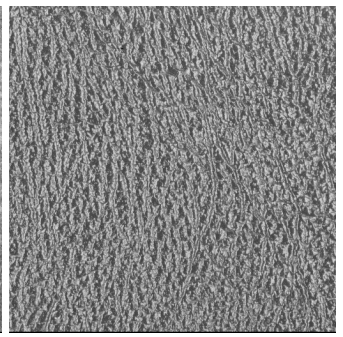

6

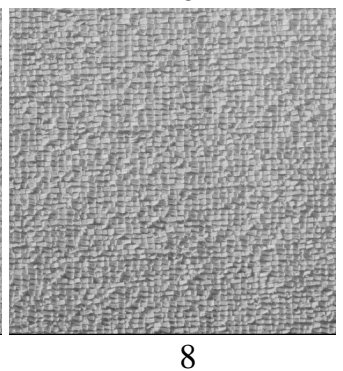

8

Fig. 1. Test texture images

\section{$4 \quad$ Results}

To study the filtering efficiency prediction, the DCT-based filter has been applied to a wide set of test images (32 test images including all images in Fig. 1) for three values of $\sigma(5,10$, and 15$)$. For each considered case, $M S E_{\text {out }} / \sigma^{2}$ or IPSNR-HVS-M have been presented as point at the corresponding scatter-plot where an argument is 
the corresponding $P_{2 \sigma}$ or $P_{2.7 \sigma}$ (see four scatter-plots in Fig. 2). We have obtained the prediction dependences using curve fitting. The fitted curves are presented in Fig. 2 by solid lines. The best obtained approximation functions for the analyzed denoising criterions are the following:

$$
\begin{gathered}
M S E_{\text {out }} / \sigma^{2}(x)=p_{1} x^{2}+p_{2} x+p_{3}, \\
\text { IPSNR - HVS - M }(x)=a \cdot \exp \left(-(x-b / b)^{2}\right),
\end{gathered}
$$

where $x$ can either be $P_{2 \sigma}$ or $P_{2.7 \sigma}$. For approximation (4), we have obtained: $p_{1}=-1.445, p_{2}=0.447, p_{3}=0.96$ for $P_{2 \sigma}$ and $p_{1}=-1.4, p_{2}=2.247$, $p_{3}=0.131$ for $P_{2.7 \sigma}$. For the approximation (5): $a=-1.445, b=0.447$, $c=0.96$ for $P_{2 \sigma}$ and $a=-1.4, b=2.247, c=0.131$ for $P_{2.7 \sigma}$. The prediction can be carried out using any probability, $P_{2 \sigma}$ or $P_{2.7 \sigma}$. In further discussions, we exploit only $P_{2 \sigma}$.

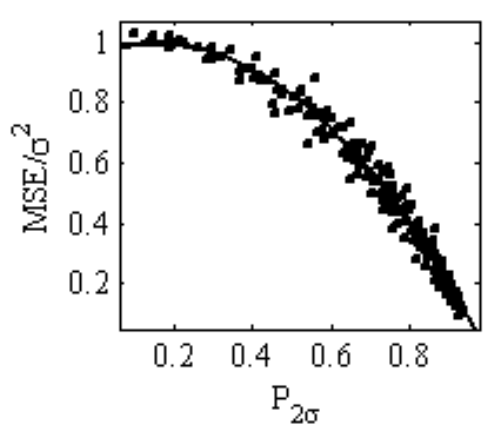

a

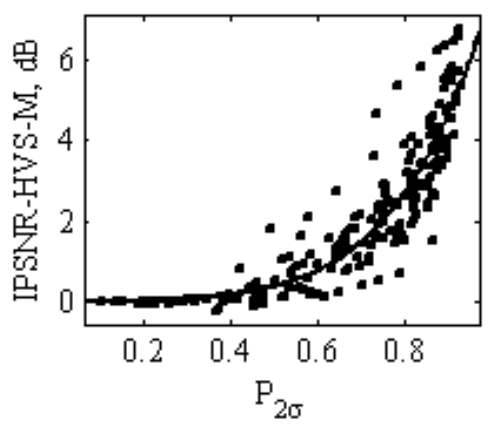

c
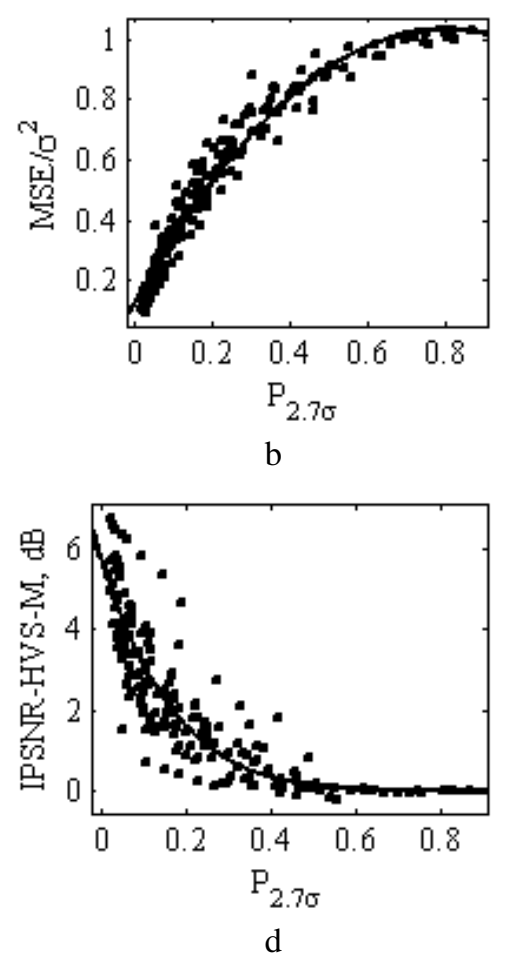

Fig. 2. Fitted curves in denoising results for $P_{2 \sigma}(a, c)$ and $P_{2.7 \sigma}(b, d)$ for the denoising criteria $\mathrm{MSE}_{\text {out }} / \sigma^{2}$ and IPSNR-HVS-M 
As it is seen, the fitted curves are close to almost all denoising results expressed by $\mathrm{MSE}_{\mathrm{out}} / \sigma^{2}$. For IPSNR-HVS-M criterion, the denoising results are more scattered. So, the curve fitting gives less precise approximation.

It is seen from Fig. 2 that if one has $P_{2 \sigma} \leq 0.5$ (or $P_{2.7 \sigma}>0.5$ ), then filtering is not efficient, i.e. $M S E_{\text {out }} / \sigma^{2}$ is close to unity and/or IPSNR-HVS-M is small (about zero). Careful analysis for the considered test images (32 images in total) has shown that this mainly happens for textural images. Note that, in fact, $P_{2 \sigma}$ and $P_{2.7 \sigma}$ quite adequately characterize "complexity" of images subject to filtering. Our proposition is that it is not worth performing image filtering if $P_{2 \sigma} \leq 0.5$ or $P_{2.7 \sigma}>0.5$.

Thus, a preliminary answer to the question "Are texture images a hard class for DCT-based denoising?" is affirmative. Then, the problem of more thorough analysis of denoising efficiency arises. Let us first express the denoising results in terms of the output MSE. Figs. 3-5 present the attained $M S E_{\text {out }}$ for the DCT and BM3D filters, $M S E_{\text {out }}$ predicted based on (8) under condition of known $\sigma^{2}$, and the lower bound $M S E_{l b}$ for the Milanfar's approach. It is seen that for $\sigma=5$ the obtained results are very close for all eight considered textures. Only for the 8-th test image the attained $M S E_{\text {out }}$ is considerably smaller than $\sigma^{2}$, i.e. denoising is rather efficient for both considered techniques.

For a higher noise level ( $\sigma=10$ and $\sigma=15$ ) the results are still close. There is almost no difference between $M S E_{\text {out }}$ for both studied denoising techniques and $M S E_{l b}$. This means that it is difficult to expect improvement of filtering efficiency for non-local family of filters applied to processing grayscale textural images. There are some textural images (\# 1 and 6), for which the noise practically cannot be removed even if its intensity is high.

The prediction analysis based on fitted curves shows that the prediction is quite accurate although it usually overestimates the filtering efficiency.

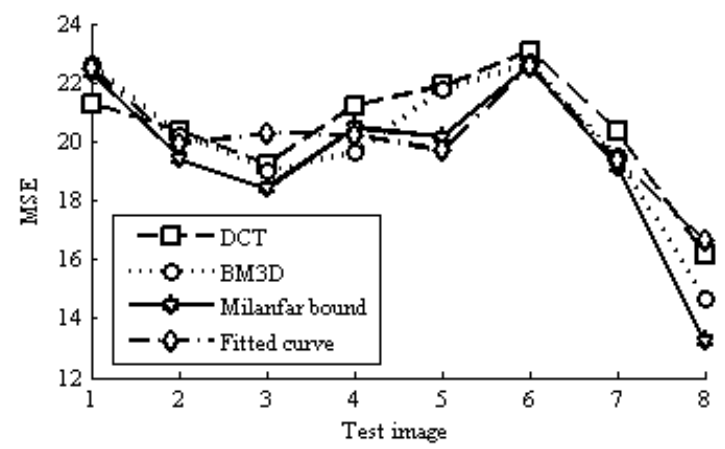

Fig. 3. Denoising results of test images expressed as output MSE for $\sigma=5$ 


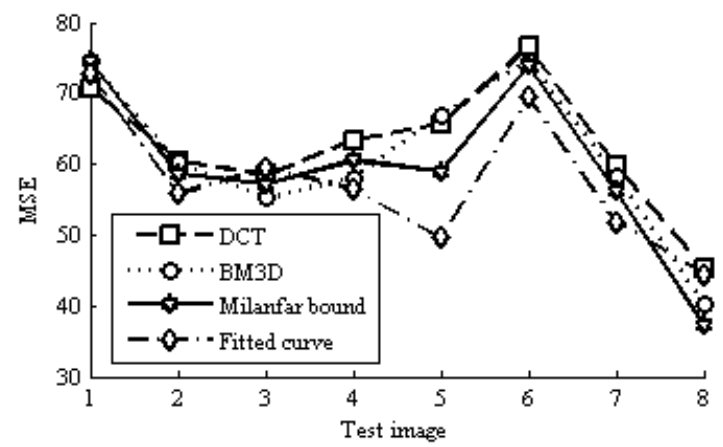

Fig. 4. Denoising results of test images expressed as output MSE for $\sigma=10$

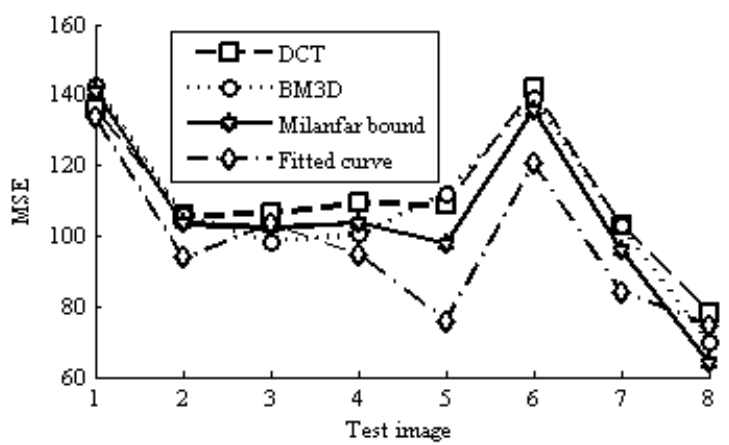

Fig. 5. Denoising results of test images expressed as output MSE for $\sigma=15$

Similarly to Figs. 3-5, denoising results characterized by IPSNR-HVS-M are presented in Figs. 6-8. Milanfar test [14] does not allow estimating PSNR-HVS-M. Thus, Fig. 6-8 present three plots for each for $\sigma$. Predicted IPSNR-HVS-M occurs to be $1 \ldots 2 \mathrm{~dB}$ larger than actual values provided by the DCT based and BM3D filters. This shows that it is desirable to improve prediction accuracy. Meanwhile, IPSNR-HVS-M almost never exceeds $1 \mathrm{~dB}$ for all considered textures and noise variance values.

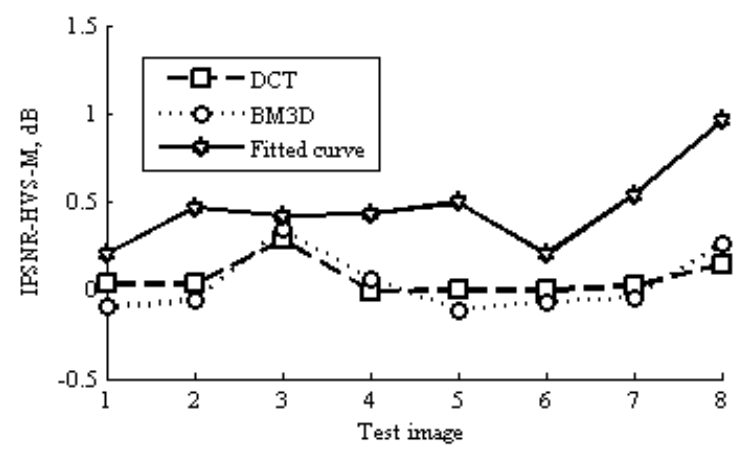

Fig. 6. Denoising results described by IPSNR-HVS-M for $\sigma=5$ 


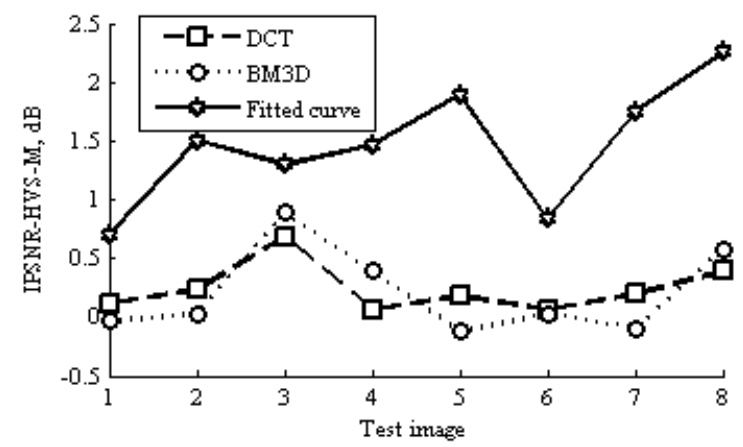

Fig. 7. Denoising results described by IPSNR-HVS-M for $\sigma=10$

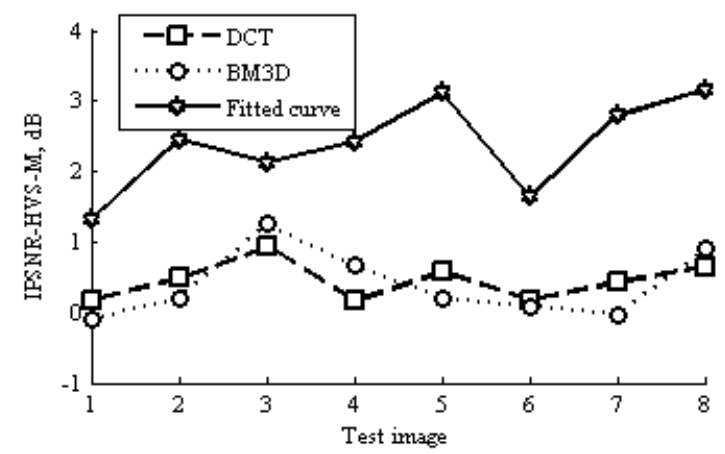

Fig. 8. Denoising results described by IPSNR-HVS-M for $\sigma=15$

This means that filtering rarely provides essential improvement of visual quality. The obtained results also show that there is no necessity to apply time consuming BM3D filter for textural regions of images since much simpler DCT-based filter performs practically at the same level.

\section{Conclusions}

It is shown that efficient denoising is not provided by the considered DCT-based filters for texture images. Meanwhile, the used local DCT and nonlocal BM3D filters provide noise suppression efficiency close to values determined by the Milanfar's bound, i.e., to potential limit for non-local approach to filtering grayscale images. Visual quality of denoised images is also not considerably better than for the original ones even if noise is intensive.

This means that, probably, it is reasonable to avoid filtering texture images (or texture fragments of images). One step towards this can deal with using prediction of denoising efficiency discussed in this paper. At the same time, additional future work concerning filtering texture images, prediction of its efficiency and design of new approaches is obviously needed. 


\section{References}

1. Haralick, R., Dori, D.: A Pattern Recognition Approach to Detection of Complex Edges. Pattern Recognition Letters 16(5), 517-529 (1995)

2. Schowengerdt, R.: Remote Sensing: Models and Methods for Image Processing, 560p. Academic Press (September 2006)

3. Cheikh, F., Cramariuc, B., Gabbouj, M.: MUVIS: A System for Content-Based Indexing and Retrieval in Large Image Databases. In: Proceedings Workshop on Very Low Bit Rate Coding, VLBV 1998, October 8-9, pp. 41-44 (1998)

4. Lukin, V., Tsymbal, O.: MM-band Radar Image Filtering with Texture Information Preservation. In: Proceedings of the Fourth International Kharkov Symposium "Physics and Engineering of Millimeter and Sub-Millimeter Waves", vol. 1, pp. 435-437 (June 2001)

5. Deledalle, C.-A., Denis, L., Tupin, F.: How to compare noisy patches? Patch similarity beyond Gaussian noise. International Journal of Computer Vision 99(1), 86-102 (2012)

6. Buades, A., Coll, A., Morel, J.M.: A non-local algorithm for image denoising. In: Computer Vision and Pattern Recognition (CVPR) IEEE Computer Society Conference, vol. 2, pp. 60-65 (2005)

7. Fevralev, D., Lukin, V., Ponomarenko, N., Abramov, S., Egiazarian, K., Astola, J.: Efficiency analysis of DCT-based filters for color image database. In: Proceedings of SPIE Conference Image Processing: Algorithms and Systems VII, vol. 7870, 12p. (2011)

8. Dabov, K.: Image and Video Restoration with Nonlocal Transform-Domain Filtering: Thesis for the degree of Doctor of Technology, Tampere, Finland, 181p. (2010)

9. Lukin, V., Oktem, R., Ponomarenko, N., Egiazarian, K.: Image filtering based on discrete cosine transform. Telecommunications and Radio Engineering 66(18), 1685-1701 (2007)

10. Sendur, L., Selesnick, I.: Bivariate Shrinkage With Local Variance Estimation. IEEE Signal Processing Letters 9(12), 4 (2002)

11. Zhou, X., Li, E., Chen, Y.-K.: Implementation of H.264 Decoder on General-Purpose Processors with Media Instructions. In: Proceeding of SPIE Conference on Image and Video Communications and Processing, vol. 5022 (2003)

12. Guo, B.-Z., Niu, L., Liu, Z.-M.: Implementation of 2-D DCT based on FPGA. In: International Conference on Image Processing and Pattern Recognition, vol. 7820, article id. 782004, p. 7 (2010)

13. Pogrebnyak, O., Lukin, V.: Wiener discrete cosine transform based image filtering. SPIE Journal of Electronic Imaging 21(4) (2012)

14. Chatterjee, P., Milanfar, P.: Is Denoising Dead? IEEE Trans. Image Processing 19(4), 895-911 (2010)

15. Lukin, V., Abramov, S., Ponomarenko, N., Egiazarian, K., Astola, J.: Image Filtering: Potential Efficiency and Current Problems. In: Proceedings of ICASSP, 4p. (2011)

16. Abramov, S., Krivenko, S., Roenko, A., Lukin, V., Djurovic, I., Chobanu, M.: Prediction of Filtering Efficiency for DCT-based Image Denoising. In: 2nd Mediterranean Conference on Embedded Computing (MECO), 4p. (2013)

17. http://sipi.usc.edu/database/database.php?volume=textures

18. Donoho, D.: Nonlinear wavelet methods for recovery of signals, densities, and spectra from indirect and noisy data. In: Proceedings Symposium Appl. Math., pp. 173-205 (1994)

19. Lukin, V., Ponomarenko, N., Egiazarian, K.: HVS-Metric-Based Performance Analysis Of Image Denoising Algorithms. In: Proceedings of EUVIP, pp. 156-161 (2011) 